\title{
Liouvillian Integrability of a Modified Michaelis-Menten Equation
}

Claudia Valls

To cite this article: Claudia Valls (2013) Liouvillian Integrability of a Modified MichaelisMenten Equation, Journal of Nonlinear Mathematical Physics 20:1, 1-8, DOI: https://doi.org/10.1080/14029251.2013.792459

To link to this article: https://doi.org/10.1080/14029251.2013.792459

Published online: 04 January 2021 


\title{
Liouvillian Integrability of a Modified Michaelis-Menten Equation
}

\author{
Claudia Valls \\ Departamento de Matemática, Instituto Superior Técnico, Av. Rovisco Pais 1049-001, Lisboa, Portugal \\ cvalls@math.ist.utl.pt \\ Received 24 May 2012 \\ Accepted 18 september 2012
}

\begin{abstract}
In this work we consider the modified Michaelis-Menten equation in biochemistry
\end{abstract}

$$
\dot{x}=-a(E-y) x+b y, \quad \dot{y}=a(E-y) x-(b+r) y, \quad \dot{z}=r y .
$$

It models the enzyme kinetics. We contribute to the understanding of its global dynamics, or more precisely, to the topological structure of its orbits by studying the integrability problem. We prove that $a=0$, or $r=0$, or $E=0$ are the unique values of the parameters for which the system is integrable, and in this case we provide an explicit expression for its first integrals.

Keywords: Liouvillian integrability, Michaelis-Menten equation, invariant algebraic surfaces, Darboux first integrals, exponential factors

2010 Mathematics Subject Classification: 34C05 34A34

\section{Introduction}

In biochemistry, the Michaelis-Menten equation models the enzime kinetics, that is, the chemical reactions that are catalysed by enzymes. In particular, it describes the rates of irreversible enzymatic reactions by relating reaction rates to the concentrations of the substrate. It was given by Michaelis and Menton in [7].

In this work we consider the Michaelis-Menten equation in the following form (see [7]):

$$
\dot{x}=-a(E-y) x+b y, \quad \dot{y}=a(E-y) x-(b+r) y, \quad \dot{z}=r y .
$$

where $x, y, z$ are the thickness of matters, $a, b, r$ are rates of response, which are nonnegative real numbers and $E$ is the quantity of enzyme, which is a constant. A number of dynamic aspects of system (1.1) have been analysed. In [3] it was discussed the existence of periodic solutions and in [4], the authors give an equation, called the Goldbeter-Koshland equation that gives the steady state solution for a chemical equilibrium modeled by the Michaelis-Menton kinetics.

Here we further contribute to the understanding of the complexity, or more precisely of the topological structure of the dynamics of system (1.1) by studying its integrability.

For the three dimensional system of differential equations the existence of one first integral reduces the complexity of its dynamics and the existence of two first integrals that are functionally independent solves completely the problem (at least theoretically) of determining its phase portraits. In general for a given differential system it is a difficult problem to determine the existence or nonexistence of first integrals. Thus, for proving our main results we shall use the information about invariant algebraic surfaces of this system. This is the basis of the so called Darboux theory of integrability, for more details see Section 2. 
C. Valls

We first consider the case in which either $a=0$, or $r=0$, or $E=0$. We start with the following result.

Theorem 1.1. The following holds for system (1.1).

(a) if $a=0$, it is integrable with the polynomial first integrals

$$
H_{1}=(b+r) x+b y \text { and } H_{2}=r x-h z .
$$

(b) if $r=0$ and $a \neq 0$, it is integrable with the polynomial first integrals

$$
H_{3}=x+y \text { and } H_{4}=z \text {. }
$$

(c) if $E=0$ and ar $\neq 0$, it is integrable with the first integrals

$$
H_{5}=x+y+z \quad \text { and } \quad H_{6}=\frac{b+a x}{a} e^{a(x+y) / r} .
$$

It is straightforward to verify that $H_{1}, H_{2}, H_{3}, H_{4}, H_{5}$ and $H_{6}$ in the statement of the theorem are first integrals of system (1.1). Therefore the proof of Theorem 1.1 will be omitted and from now on we consider the case in which $\operatorname{ar} E \in \mathbb{R} \backslash\{0\}$. We note that the first integrals $H_{1}, H_{2}, H_{3}, H_{4}, H_{5}$ and $H_{6}$ are all Liouvillian.

When $\operatorname{ar} E \in \mathbb{R} \backslash\{0\}$ we have that

$$
H=x+y+z
$$

is a first integral of system (1.1). Therefore, setting $H=h, h \in \mathbb{R}$ we can rewrite system (1.1) as the following system in the plane $(z=H-x-y)$ :

$$
\dot{x}=-a(E-y) x+b y, \quad \dot{y}=a(E-y) x-(b+r) y .
$$

From now on instead of working with system (1.1) we will work with system (1.2). In particular, we introduce the variables $X=x+y, Y=y$. We have that

$$
\dot{X}=-r Y, \quad \dot{Y}=-(b+r+a E) Y+a E X-a Y X+a Y^{2} .
$$

The following theorem is the main result of this paper. In particular, it states the non-existence of Liouvillian first integrals for system (1.1) when $\operatorname{ar} E \in \mathbb{R} \backslash\{0\}$.

Theorem 1.2. The following statements hold for system (1.3) with arE $\in \mathbb{R} \backslash\{0\}$ :

(a) It does not admit any polynomial first integral;

(b) It has an irreducible Darboux polynomial with non-zero cofactor if and only if $b=0$ and in this case the Darboux polynomial is $f=X-Y$ and the cofactor is $-a(E-Y)$.

(c) Its only exponential factor is $e^{X}$ with the cofactor $-r Y$;

(d) It does not admit any Darboux first integral.

(e) It does not admit any Liouvillian first integral.

As far as we know the Liouvillian first integral of some multi-parameter family of planar polynomial differential systems has been classified for very few families of systems (see for instance $[1,6,8])$.

The paper is organised as follows. In Section 2 we introduce some basic definitions and results related to the Darboux theory of integrability that we shall need in order to prove one of our main results. In Section 3 we prove Theorem 1.2. 


\section{Preliminary results}

During recent years the interest in the study of integrability of differential equations has attracted much attention. Darboux theory of integrability plays a central role in the integrability of the polynomial differential models. It gives a sufficient condition for the integrability inside the family of Darboux functions. More precisely, the significance of this method is that we can compute Darboux first integrals by knowing a sufficient number of algebraic invariant surfaces (the so-called Darboux polynomials) and of the so-called exponential factors. We would like to highlight that it works for real or complex polynomial ordinary differential equations. The study of complex invariant algebraic curves is necessary for obtaining all the real first integrals of a real polynomial differential equation, for more details see [5].

We associate to system (1.1) the following vector field

$$
\mathfrak{X}=-r Y \frac{\partial}{\partial X}+\left(-(b+r+a E) Y+a E X-a Y X+a Y^{2}\right) \frac{\partial}{\partial Y} .
$$

Let $U \subset \mathbb{R}^{2}$ be an open subset. We say that the non-constant function $H: U \rightarrow \mathbb{R}$ is a first integral of the polynomial vector field (2.1) associated to system (1.3), if $H(X(t), Y(t))=$ constant for all values of $t$ for which the solution $(X(t), Y(t))$ of $\mathfrak{X}$ is defined on $U$. Clearly $H$ is a first integral of $\mathfrak{X}$ on $U$ if and only if $\mathfrak{X} H=0$ on $U$. When $H$ is a polynomial we say that $H$ is a polynomial first integral.

Let $h=h(X, Y) \in \mathbb{C}[X, Y]$ be a non-constant polynomial. We say that $h=0$ is an invariant algebraic surface of the vector field $\mathfrak{X}$ in (2.1) if it satisfies $\mathfrak{X} h=K h$, for some polynomial $K=$ $K(X, Y) \in \mathbb{C}[X, Y]$, called the cofactor of $h$. Note that $K$ has degree at most 1 . The polynomial $h$ is called a Darboux polynomial, and we also say that $K$ is the cofactor of the Darboux polynomial $h$. We note that a Darboux polynomial with a zero cofactor is a polynomial first integral.

The following proposition provides a basic property of the Darboux polynomials and it states that to study Darboux polynomials it is sufficient to study the irreducible ones.

Proposition 2.1. Let $f$ be a polynomial and $f=\prod_{j=1}^{s} f_{j}^{\alpha_{j}}$ its decomposition into irreducible factors in $\mathbb{C}\left[x_{1}, \ldots, x_{n}\right]$. Then, $f$ is a Darboux polynomial if and only if all the $f_{j}$ are Darboux polynomials. Moreover, if $K$ and $K_{j}$ are the cofactors of $f$ and $f_{j}$, then $K=\sum_{j=1}^{s} \alpha_{j} K_{j}$.

Let $g, h \in \mathbb{C}[X, Y]$ be coprime. We say that a non-constant function $E=e^{h / g}$ is an exponential factor of the vector field $\mathfrak{X}$ given in (2.1) if it satisfies $\mathfrak{X} E=L E$, for some polynomial $L=L(X, Y) \in$ $\mathbb{C}[X, Y]$, called the cofactor of $E$ and having degree at most 1 . Note that this relation is equivalent to

$$
-r Y \frac{\partial(g / h)}{\partial X}+\left(-(b+r+a E) Y+a E X-a Y X+a Y^{2}\right) \frac{\partial(g / h)}{\partial Y}=L .
$$

For a geometrical and algebraic meaning of the exponential factors see [2].

A first integral $G$ of system (1.1) is called of Darboux type if it is of the form

$$
G=f_{1}^{\lambda_{1}} \cdots f_{p}^{\lambda_{p}} E_{1}^{\mu_{1}} \cdots E_{q}^{\mu_{q}},
$$


C. Valls

where $f_{1}, \ldots, f_{p}$ are Darboux polynomials, $E_{1}, \ldots, E_{q}$ are exponential factors and $\lambda_{j}, \mu_{k} \in \mathbb{C}$ for $j=1, \ldots, p, k=1, \ldots, q$. For more information on the Darboux theory of integrability see, for instance, [5] and the references therein.

For a proof of the next proposition see [2].

Proposition 2.2. The following statements hold:

(a) If $E=e^{g / h}$ is an exponential factor for the polynomial system (1.1) and $h$ is not a constant polynomial, then $h=0$ is an invariant algebraic curve.

(b) Eventually $e^{g}$ can be an exponential factor, coming from the multiplicity of the infinite invariant straight line.

A Liouvillian first integral is a first integral $H$ which is a Liouvillian function, that is, roughly speaking which can be obtained "by quadratures" of elementary functions. For a precise definition see [9]. The study of the Liouvillian first integrals is a classical problem of the integrability theory of the differential equations which goes back to Liouville, see for details again [9].

A non-constant complex function $R: \mathbb{C}^{2} \rightarrow \mathbb{C}$ is an integrating factor of the polynomial vector field $\mathfrak{X}$ in (2.1) on $U$, if one of the following three equivalent conditions holds

$$
\frac{\partial(R P)}{\partial X}=-\frac{\partial(R Q)}{\partial Y}, \quad \operatorname{div}(R P, R Q)=0, \quad X R=-R \operatorname{div}(P, Q),
$$

on $U$ and where $P=-r Y$ and $Q=-(b+r+a E) Y+a E X-a Y X+a Y^{2}$. As usual the divergence of the vector field $\mathfrak{X}$ is given by

$$
\operatorname{div}(P, Q)=\frac{\partial P}{\partial X}+\frac{\partial Q}{\partial Y}
$$

To prove the results related with Liouvillian first integrals we use the following result proved in [9].

Theorem 2.1. The polynomial differential system (1.3) has a Liouvillian first integral if and only if it has an integrating factor of Darboux type.

We also need the following well-known statement.

Theorem 2.2. Suppose that the polynomial vector field (1.3) has p invariant Darboux polynomials with cofactors $K_{i}$, for $i=1, \ldots, p$ and $q$ exponential factors with cofactors $L_{j}$, for $j=1, \ldots, q$. Then there exist $\lambda_{i}, \mu_{j} \in \mathbb{C}$ not all zero such that

$$
\sum_{i=1}^{p} \lambda_{i} K_{i}+\sum_{j=1}^{q} \mu_{j} L_{j}=-\operatorname{div}(P, Q)
$$

if and only if the function of Darboux type (2.2) is an integrating factor of the vector field $\mathfrak{X}$.

\section{Proof of Theorem 1.2}

We separate the proof of Theorem 1.2 into different propositions.

Proposition 3.1. System (1.3) with arE $\in \mathbb{R} \backslash\{0\}$ does not admit a polynomial first integral. 
Proof. Let $h$ be a polynomial first integral of system (1.3). Then it satisfies

$$
-r Y \frac{\partial h}{\partial X}+\left(-(b+r+a E) Y+a E X-a Y X+a Y^{2}\right) \frac{\partial h}{\partial Y}=0 .
$$

Without loss of generality we can write

$$
h=\sum_{j=1}^{n} h_{j}(X, Y)
$$

where each $h_{j}=h_{j}(X, Y)$ is a homogeneous polynomial of degree $j$ and we assume that $h_{n} \neq 0$.

Computing the terms of degree $n+1$ in (3.1) we get

$$
a Y(Y-X) \frac{\partial h_{n}}{\partial Y}=0 .
$$

Solving this differential equation we obtain $h_{n}=h_{n}[X]$. Since $h_{n} \neq 0$ is a homogeneous polynomial of degree $n \geq 1$, we conclude that $h_{n}=\alpha_{n} X^{n}$, with $\alpha_{n} \in \mathbb{C}$.

Computing the terms of degree $n$ in (3.1) we get

$$
a Y(Y-X) \frac{\partial h_{n-1}}{\partial Y}-r Y n \alpha_{n} X^{n-1}=0 .
$$

Evaluating it on $X=Y$ it yields $-r n \alpha_{n} Y^{n}=0$, which implies $\alpha_{n}=0$, and this is not possible. This concludes the proof of the proposition.

Proposition 3.2. System (1.3) with arE $\in \mathbb{R} \backslash\{0\}$ admits an irreducible Darboux polynomial $f$ with nonzero cofactor $K$ if and only if $b=0$ and in this case $f=X-Y$ and $K=-a(E-Y)$.

Proof. Let $h$ be an irreducible Darboux polynomial of system (1.1) with nonzero cofactor $K$, where $K=\alpha_{0}+\alpha_{1} X+\alpha_{2} Y$, with $\alpha_{i} \in \mathbb{C}$ for $i=0,1,2$ not all zero.

Then $h$ satisfies

$$
-r Y \frac{\partial h}{\partial X}+\left(-(b+r+a E) Y+a E X-a Y X+a Y^{2}\right) \frac{\partial h}{\partial Y}=\left(\alpha_{0}+\alpha_{1} X+\alpha_{2} Y\right) h .
$$

It is easy to see by direct computations that if $h$ has degree one then $h$ is different from zero if and only if $b=0$ and in this case $h=Y-X$. Now we assume that $h$ is irreducible and has degree greater or equal to two. Thus we decompose $h$ as a sum of homogeneous polynomials similarly as in (3.2), where $n \geq 2$ and $h_{n} \neq 0$.

Computing the terms of degree $n+1$ in (3.5) we get

$$
a Y(Y-X) \frac{\partial h_{n}}{\partial Y}=\left(\alpha_{1} X+\alpha_{2} Y\right) h_{n}
$$

Solving this linear differential equation we obtain

$$
h_{n}=K_{n}(X) Y^{-\alpha_{1} / a}(Y-X)^{\alpha_{2} / a+\alpha_{1} / a},
$$

where $K_{n}$ is a function in the variable $X$. Since $h_{n}$ is a homogeneous polynomial of degree $n$ we must have $\alpha_{1}=0$ and $\alpha_{2}=$ am with $m \in \mathbb{N}$. Hence

$$
h_{n}=c_{n} X^{n-m}(Y-X)^{m},
$$

where $c_{n} \in \mathbb{C} \backslash\{0\}$ (since $h_{n} \neq 0$ ). 
C. Valls

Computing the terms of degree $n$ in (3.5) we get

$$
a Y(Y-X) \frac{\partial h_{n-1}}{\partial Y}+(a E X-(b+r+a E) Y) \frac{\partial h_{n}}{\partial Y}-r Y \frac{\partial h_{n}}{\partial X}=a m Y h_{n-1}+\alpha_{0} h_{n} .
$$

Substituting (3.6) into equation (3.7) and solving it with respect to $h_{n-1}$ we obtain

$$
\begin{aligned}
h_{n-1}= & c_{n}(X)(X-Y)^{m}-\frac{c_{n}}{a} X^{n-m-1}(Y-X)^{m-1}(b m X \\
& +(X-Y)\left(\left(\alpha_{0}+a E m-m r+n r\right) \log (X-Y)-\left(\alpha_{0}+a E m\right) \log (-Y)\right) .
\end{aligned}
$$

Since $\operatorname{ar} E \neq 0, c_{n} \neq 0$ and $h_{n-1}$ is a homogeneous polynomial of degree $n-1$, we get the conditions

$$
m=n \quad \text { and } \quad \alpha_{0}=-a E n .
$$

Hence

$$
h_{n}=c_{n}(Y-X)^{n} \quad \text { and } \quad h_{n-1}=-\frac{c_{n} b n}{a}(X-Y)^{n-1} .
$$

Now we consider two cases.

Case 1: $b \neq 0$. Computing the terms of degree $n-1$ in (3.5) we get

$$
a Y(Y-X) \frac{\partial h_{n-2}}{\partial Y}+(a E X-(b+r+a E) Y) \frac{\partial h_{n-1}}{\partial Y}-r Y \frac{\partial h_{n-1}}{\partial X}=a n Y h_{n-2}-a E n h_{n-1} .
$$

Substituting (3.8) into equation (3.9) and solving it with respect to $h_{n-2}$ we obtain

$$
\begin{aligned}
h_{n-2}= & \frac{c_{n} b n}{2 a X^{2}}(Y-X)^{n-2}(X(b(n-1) X+2 a E(X-Y)) \\
& \left.+2 a E(X-Y)^{2}(-\log (X-Y)+\log (-Y))\right)+K_{n-2}(X)(X-Y)^{n} .
\end{aligned}
$$

Since $h_{n-2}$ is a homogeneous polynomial of degree $n-2$ and $a E b \neq 0$ we get a contradiction. Hence this case is not possible.

Case 2: $b=0$. In this case $h_{n}=c_{n}(Y-X)^{n}$ and $h_{n-1}=0$. We consider $\bar{h}$ to be the restriction of $h$ to $Y=X$. If we write $\bar{h}$ in sum of its homogeneous parts we get that $\bar{h}=\sum_{j=0}^{n} \bar{h}_{j}$ where each $\bar{h}_{j}=\bar{h}_{j}(X)$ is a homogeneous polynomial of degree $j$ and $\bar{h}_{j}$ is the restriction of $h_{j}$ to $Y=X$. Since $h$ is irreducible we must have $\bar{h} \neq 0$. However we have that $\bar{h}_{n}=0$ and then $\bar{h}=0$, which is not possible. This completes the proof of the proposition.

Proposition 3.3. The only exponential factors of system (1.3) with arE $\in \mathbb{R} \backslash\{0\}$ are $e^{X}$ with the cofactor $-r Y$.

Proof. We consider two different cases.

Case 1: $b \neq 0$. In this case it follows from Proposition 2.2 that we can write $E=e^{g}$ and $g$ satisfies

$$
-r Y \frac{\partial g}{\partial X}+\left(-(b+r+a E) Y+a E X-a Y X+a Y^{2}\right) \frac{\partial g}{\partial Y}=\alpha_{0}+\alpha_{1} X+\alpha_{2} Y,
$$

where $\alpha_{i} \in \mathbb{C}$, for $i=0,1,2$ are not all zero.

We first prove that $g$ is a polynomial of degree two. We proceed by contradiction. Assume that $g$ is polynomial of degree $n \geq 3$. We write it as a sum of its homogeneous parts as in equation (3.2) 
with $h_{j}$ replaced by $g_{j}$. Since the right-hand side of equation (3.10) has degree at most one, computing the terms of degree $n+1$ in equation (3.10) we get

$$
a Y(Y-X) \frac{\partial g_{n}}{\partial Y}=0
$$

which is equation (3.3) replacing $h_{n}$ by $g_{n}$. Then the arguments used in the proof of Proposition 3.1 imply that $g_{n}=\alpha_{n} X^{n}$ with $\alpha_{n} \in \mathbb{C}$.

Now computing the terms of degree $n \geq 3$ in (3.10) and taking into account that the right-hand side of (3.10) has degree one, we get equation

$$
-r Y \frac{\partial g_{n-1}}{\partial X}+(-(b+r+a E) Y+a E X) \frac{\partial g_{n-1}}{\partial Y}+a Y(Y-X) \frac{\partial g_{n}}{\partial Y}=0,
$$

which is equation (3.4) with $h_{n}$ replaced by $g_{n}$ and $h_{n-1}$ replaced by $g_{n-1}$. The arguments used in the proof of Proposition 3.1 imply that $g_{n}=0$. Then we have that $g_{n}=0$ for $n \geq 3$, and thus, $g$ is a polynomial of degree at most two satisfying (3.10). Solving now (3.10) we get that $g$ must be $X$ with cofactor $L=-r Y$ and the proposition follows.

Case 2: $b=0$. In this case it follows from Proposition 2.2 that we can write $E=e^{g /(Y-X)^{n}}$ with $n \in \mathbb{N} \cup\{0\}$ and $g$ being coprime with $Y-X$ (when $n>0$ ). Furthermore, $g \in \mathbb{C}[X, Y]$ satisfies

$$
\begin{aligned}
& -r Y \frac{\partial g}{\partial X}+\left(-(b+r+a E) Y+a E X-a Y X+a Y^{2}\right) \frac{\partial g}{\partial Y} \\
& =n a(E-Y) g+\left(\alpha_{0}+\alpha_{1} X+\alpha_{2} Y\right)(Y-X)^{n},
\end{aligned}
$$

where $\alpha_{i} \in \mathbb{C}$, for $i=0,1,2$ are not all zero. If $n=0$ then $E=e^{g}$ and $g$ satisfies equation (3.10) and proceeding as in Case 1 we get that $g=X$ with the cofactor $L=-r Y$. Now we assume that $n>0$. In this case if we denote by $\bar{g}=\bar{g}(X)$ the restriction of $g$ to $Y=X$ we get that $\bar{g} \neq 0$ (otherwise $g$ would not be coprime with $Y-X$ ) and satisfies

$$
-r X \frac{\partial \bar{g}}{\partial X}=n a(E-X) \bar{g}
$$

Solving it we get $\bar{g}=K e^{a n X / r} X^{-a d n / r}, K \in \mathbb{C}$. Since $\bar{g}$ must be a polynomial and $a n r \neq 0$ we must have $K=0$ and then $\bar{g}=0$, a contradiction. This case is not possible. This concludes the proof of the proposition.

\subsection{Proof of Theorem 1.2}

Statements (a), (b) and (c) in the theorem follow directly from Propositions 3.1, 3.2 and 3.3, respectively. In what follows we prove statements (d) and (e) by contradiction.

We first prove statement (d). Assume that $G$ is a first integral of Darboux type. Then in view of its definition in (2.2) and taking into account Propositions 3.1, 3.2 and 3.3, $G$ must be of the form

$$
G= \begin{cases}e^{\mu_{1} X}, \quad \text { with } \quad \mu_{1} \in \mathbb{C}, & \text { if } b \neq 0, \\ (Y-X)^{\lambda_{1}} e^{\mu_{1} X}, \quad \text { with } \quad \lambda_{1}, \mu_{1} \in \mathbb{C}, & \text { if } b=0 .\end{cases}
$$


Since $G$ is a first integral it must satisfy $\mathfrak{X} G=0$, that is,

$$
\begin{aligned}
0 & =\mathfrak{X} G=-r Y \frac{\partial G}{\partial X}+(-(b+r+a E) Y+a E X+a Y(Y-X)) \frac{\partial G}{\partial Y} \\
& = \begin{cases}-r \mu_{1} Y G, & \text { if } b \neq 0, \\
\left(-a \lambda_{1}(E-Y)-r \mu_{1} Y\right) G, & \text { if } b=0 .\end{cases}
\end{aligned}
$$

Hence, since $r \neq 0$ we get that if $b \neq 0$ then $\mu_{1}=0$ and thus $G$ is a constant. Moreover, since $a E \neq 0$ if $b=0$ we must have $\lambda_{1}=0$ and then also $\mu_{1}=0$ and thus $G$ is also a constant. In both cases we get that $G$ is a constant, in contradiction with the fact that $G$ is a first integral. This completes the proof of statement (d).

Now we prove statement (e). By Theorem 2.1 in order that system (1.3) has a Liouvillian first integral it must have an integrating factor of Darboux type (see (2.2)). From Theorem 2.2 system (1.3) has an integrating factor of Darboux type if and only if

$$
b+r+a E+a X-2 a Y= \begin{cases}-r \mu_{1} Y, & \text { if } b \neq 0, \\ -a \lambda_{1}(E-Y)-r \mu_{1} Y, & \text { if } b=0,\end{cases}
$$

with $\lambda_{1}, \mu_{1} \in \mathbb{C}$. Since $a \neq 0$ the equality in (3.11) is not possible because the right-hand side is independent of $X$. This ends the proof of the theorem.

\section{Acknowledgements}

Partially supported by FCT through CAMGDS, Lisbon.

\section{References}

[1] L. CAiró, H. Giacomini AND J. Llibre, Liouvillian first integrals for the planar Lotka-Volterra system, Rend. Circ. Mat. Palermo 52 (2003), 389-418.

[2] C. Christopher, J. Llibre, and J.V. Pereira, Multiplicity of invariant algebraic curves in polynomial vector fields, Pacific J. Math. 229 (2007), 63-117.

[3] L. Dai, On the existence, uniqueness and global asymptotic stability of the periodic solution of the modificed Michaelis-Menton Mechanis, J. Diff. Equations, 31 (1979), 391-417.

[4] A. Goldbeter and D. Koshland, An amplified sensitivity arising from covalent modification in biological systems, Proc. Natl. Acad. Sci. U.S.A, 78 (1981), 6840-6844.

[5] J. Llibre, Integrability of polynoial differential systems, Handbook of Differential Equations, Ordinary Differential Equations, Eds. A. Cañada, P. Drabek and A. Fonda, 1 (2004), 437-533.

[6] J. LliBRE AND C. VALLS, Liouvillian first integrals for Liénard polynomial differential systems, Proc. Amer. Math. Soc. 138 (2010), 3229-3239.

[7] L. Michaelis and M. Menten, Biochem. Z. 49 (1913), 333.

[8] J. Moulin OllaGNieR, Liouvillian Integration of the Lotka-Volterra system, Qual. Theory Dyn. Syst. 2 (2001), 307-358.

[9] M. F. Singer, Liouvillian first integrals of differential systems, Trans. Amer. Math. Soc. 333 (1992), 673-688. 